\title{
Confocal Raman spectroscopy: determination of natural moisturizing factor profile related to skin hydration
}

\author{
Ana Paula Teixeira, João Lucas Rangel, Leandro José Raniero, Maira Gaspar Tosato, Priscila Pereira \\ Fávero, Airton Abrahão Martin*
}

\begin{abstract}
Introduction: Skin health and skin care to reduce the effects of aging are the main interests of many researchers. The skin is very important because it protects the body from various effects of the external environment, and studies of the largest organ of the human body have been conducted since antiquity. In skin, aging effects are severe enough to promote changes in cell structure and biochemical composition. In this study, we quantitatively analyzed the water content and natural moisturizing factor of human facial skin in vivo and in real time by confocal Raman spectroscopy. This non-invasive technique is capable of providing detailed information on the biochemical composition at different depth profiles in the skin. Methods: We studied 10 volunteers, phototype II (40 and 50 years old), using a confocal Raman system to examine the skin surface down to $25 \mu \mathrm{m}$. Raman spectra were obtained before product use (T0), and after 30 days of continuous use of cosmetics (T30). Results: The results show a significant increase of $6.4 \%$ in water content in the surface layer of the facial skin after the cosmetic use. The amounts of natural moisturizing factor (NMF) compounds were also increased. Urocanic acid underwent a greater change in relation to carboxylic acid pyrrolidone, with a $38.5 \%$ increase in the stratum corneum. Conclusion: Confocal Raman spectroscopy identified changes in the biochemical composition of the superficial layers of the epidermis, which suggests the anti-aging efficacy of the formulation.
\end{abstract}

Keywords Skin aging, Cosmetics, Confocal Raman spectroscopy, NMF.

\section{Introduction}

The skin, the interface between the biological body and the environment, is divided into three layers: the epidermis, dermis, and subcutaneous tissue. The epidermis is composed of stratified epithelium, which is regenerated continuously, and it varies in thickness from $40 \mu \mathrm{m}$ to $1 \mathrm{~mm}$ for different body regions. Epidermal cells are first keratinocytes, which during maturation, migrate upward to the surface of the skin, forming a layer approximately 10 to $15 \mu \mathrm{m}$ thick, denominated the stratum corneum. In this layer, keratinocytes become corneocytes (hydrophilic) and are stacked into layers interspersed with an array of intercellular lipid structures (hydrophobic). These intercellular lipid structures are composed of ceramides, cholesterol, and fatty acids, which act as a barrier against dehydration (Grayson and Elias, 1982). The degree of organization of lipids is of utmost importance in preventing transepidermal water loss (TEWL) (Costa, 2012). Below the epidermis is the dermis, which has a thickness ranging from 0.5 to $4 \mathrm{~mm}$ and is comprised primarily of collagen and elastin (Caspers et al., 2003).

The skin aging process is quite complex, as it is the sum of a series of changes related to intrinsic and extrinsic factors. During the skin aging process, biochemical, cellular, and tissue changes mainly result in a decrease in the dermal-epidermal interface. These changes affect the transport of nutrients between the layers of the skin, causing a decrease in the extracellular matrix and the fragmentation of elastic fibers (Votano et al., 2004), while promoting the reduction of collagen synthesis by impairing the activity of fibroblasts (Chung et al., 2001). Biochemical and histological skin alterations cause anatomical transformations such as melanosis, the formation of wrinkles, actinic keratoses, loss of elasticity, decreased hydration, and photodamage (Bagatin, 2008).

Studies of the biomechanical properties of skin and the influence of active components are usually performed using invasive techniques that remove the skin tissue for ex vivo analysis. Currently, scanometry is the most common method for ex vivo study in the first sublayers of the skin, performed by successive applications of adhesive films (Alberti et al., 2001). However, the standardization of applied force is difficult with this method, resulting in changes in the thickness profile of the removed skin. Some non-invasive methods, such as measurement of 
electrical properties, are employed in the evaluation of skin hydration. Studies on skin hydration have bee conducted by measures of skin capacitance, electrical conductance, transepidermal water loss (TEWL), and pH electrode (Djabri et al., 2012; Fraser et al., 2012). Such electrical measurements provide qualitative information on changes in the general characteristics of the skin, but they do not measure changes in its molecular composition.

Due to the limitations of conventional techniques, various studies have been performed using noninvasive optical techniques. Among these techniques, in vivo confocal Raman spectroscopy is a powerful method to identify and characterize changes in biochemical structures and molecular conformations resulting from aging processes in living tissues in real time (Oliveira et al., 2012; Tosato et al., 2012).

Given the effects of the skin aging process and the complexity in understanding all the agents involved, the objective of this study was to identify the Raman spectral regions where the greatest biochemical changes occur in the skin, in the absence and presence of a cosmetic anti-aging formulation. In this work, we analyzed the water and natural moisturizing factor (NMF) content. The NMF is found exclusively in the stratum corneum and comprises amino acids and their derivatives, such as inorganic ions. The NMF forms a complex medium responsible for maintaining the optimum quantity of water in the skin (Rawlings and Harding, 2004). Analyses were performed in different layers of the skin by in vivo confocal Raman spectroscopy to understand the possible changes and relate these changes to the use of the product.

\section{Methods}

This work was developed at the Laboratory of Biomedical Vibrational Spectroscopy at Universidade do Vale do Paraíba, following the Guidelines and Standards for Research Involving Humans, according to Resolution 196/96 of the National Health Council. The study was approved by the Research Ethics Committee (CEP) of the Institute for Research and Development of Univap, under protocol number H48/ CEP/2008. All subjects signed a consent form before the start of the study and received information about study procedures.

\section{Selection of volunteers}

The study comprised 10 volunteers with phototype II, classified according to skin color and reaction to sun exposure (white skin, hardly tans, burns easily - sensitive), ranging in age between 40 and 50 years. The participants all had wrinkles and fine lines on the skin of their face and did not frequently use products to reduce aging marks, or routinely use makeup. The following exclusion criteria were used for the study: presence of skin disease, pregnancy or lactation, irritation or cosmetic sensitivity to products with anti-aging active ingredients, and the start of or changes in medication.

\section{In vivo experimental methodology}

To evaluate the possible biochemical changes in the components of facial skin due to use of the product, the study was divided into two stages, T0 and T30. The first measurement, $\mathrm{T} 0$, was performed after seven days without the use of any face cosmetic products by the volunteers. After initial measurements, the volunteers were instructed to use the product continuously for 30 consecutive days. We recommended the application of the cosmetic twice a day (morning and afternoon), across the face. The final measurement, T30, was measured after 30 days.

Before starting spectroscopic measurements of the skin, the volunteers used water to wash the area to be measured and waited for $30 \mathrm{~min}$ in an air-conditioned environment at $23 \pm 1{ }^{\circ} \mathrm{C}$ and relative humidity of 40 $50 \%$. The analyses were performed at the right side of the periorbicular region of the face, and Raman spectra were measured with steps of $5 \mu \mathrm{m}$ from the surface of the skin to $25 \mu \mathrm{m}$ in depth. Raman data were taken at 2 different points, $\mathrm{P} 1$ and $\mathrm{P} 2$, separated by a horizontal distance of approximately $30 \mu \mathrm{m}$. To assess the variance of the results in P1 and P2, two spectral acquisitions were taken. Each acquisition was divided into two spectral regions, classified as high and low frequency, totaling eight readings for each depth for a single volunteer.

\section{Raman measurements}

Non-invasive analyses were performed by confocal Raman spectroscopy using a system from Rivers Diagnosis (Model 3510), with laser excitation at $785 \mathrm{~nm}$ and $671 \mathrm{~nm}$ and power at the sample of $25 \mathrm{~mW}$ and $10.6 \mathrm{~mW}$, respectively. The Raman data were collected by a CCD detector. We analyzed the spectral fingerprint $\left(400-1800 \mathrm{~cm}^{-1}\right)$ and high frequency region (2800-3600 $\left.\mathrm{cm}^{-1}\right)$ at the following depths: $0,5,10,15,20,25 \mu \mathrm{m}$. The integration time was 10 seconds for each depth.

\section{Data processing}

The concentration of the stratum corneum components at different depths was calculated semi-quantitatively in relation to keratin concentration using the software Skin Tools ${ }^{\circledR}$. This method is well described by Caspers et al. (2001). 
The concentration profile of water in skin (water content) as a function of depth was determined in the high-frequency Raman spectra region. This was performed using the ratio between the integrated area of the keratin peaks (between 2910 and $2960 \mathrm{~cm}^{-1}$ ) and water molecule peaks (between 3350 and $3550 \mathrm{~cm}^{-1}$ ) resulting from $-\mathrm{CH}_{3}$ stretching vibrations and $\mathrm{OH}$ stretch. The integration was performed assuming a linear baseline between 2600 and $3800 \mathrm{~cm}^{-1}$ (Bergholt et al., 2013; Caspers et al., 2000; De Carvalho et al., 2011). The water content ( $\%$ by mass) is expressed in grams of water per $100 \mathrm{~g}$ of skin tissue and was calculated from the ratio of the water-band protein. The first derivative of the average profiles of the curves for water concentration (T0 and T30) was used to determine the approximate thickness of the stratum corneum, indicated by the minimum $x$-value point. Beyond this value, the rate of change became zero.

For the low-frequency region of the Raman spectrum, known as the fingerprint region, the calculation of the overall NMF was performed. This region comprises amino acids such as PCA (pyrrolidone carboxylic acid), urocanic acid (AUC), lactate, urea, serine, glycine, arginine, citrulline, ornithine, alanine, histidine, and phenylalanine. PCA and AUC are considered the main components of NMF. Therefore, they were chosen for the analysis of NMF in the skin. The semi-quantitative determination of the molecular concentration of each amino acid for different depths was performed using the least square fit of the spectra and compared to the pure spectra of each constituent (Caspers et al., 2001).

\section{Results}

\section{Determination of water concentration}

Figure 1 shows the average confocal Raman spectra of $\mathrm{T} 0$ and $\mathrm{T} 30$ at different depths in the high frequency region. Compared to $\mathrm{T} 0$, there is a notable increase in the intensity of the band centered at $3420 \mathrm{~cm}^{-1}$ for T30 from the surface to $20 \mu \mathrm{m}$ in depth. The calculated water concentration profile is shown in Figure 2. The spectral region at $2800-3000 \mathrm{~cm}^{-1}$, assigned to the stretch vibrations of $\mathrm{CH}$ and $\mathrm{CH} 3$ in keratin, is better discriminated for T30, at up to 5 micrometers in depth.

The calculated values (Figure 2) indicate that the water content at the skin surface is approximately $35 \%$. The water content gradually increases with depth until reaching a constant value equal to $70 \%$ for T30 near $15 \mu \mathrm{m}$ (intersection of the gray line).

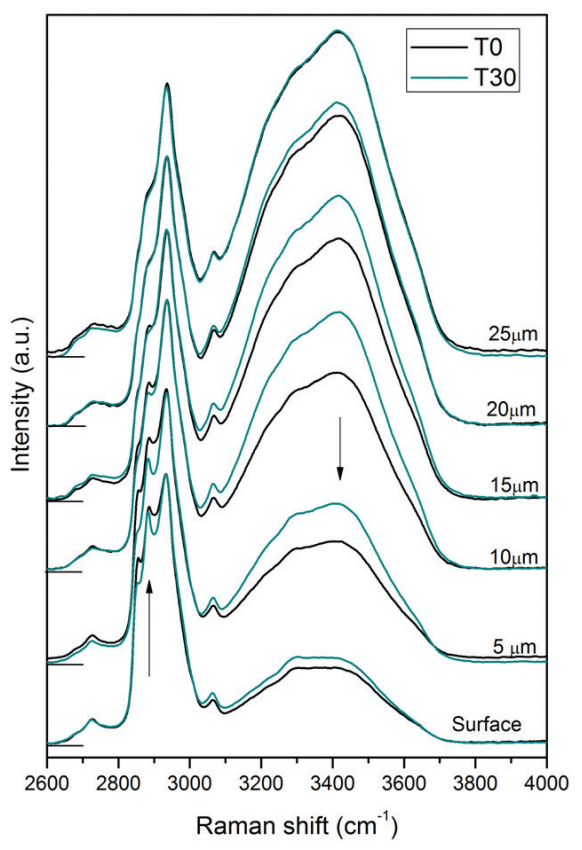

Figure 1. Average Raman spectra of the high-frequency region (2600 to $4000 \mathrm{~cm}^{-1}$ ) for T0 (black) and T30 (gray) for different depths. For clarity, the spectra have been shifted vertically.

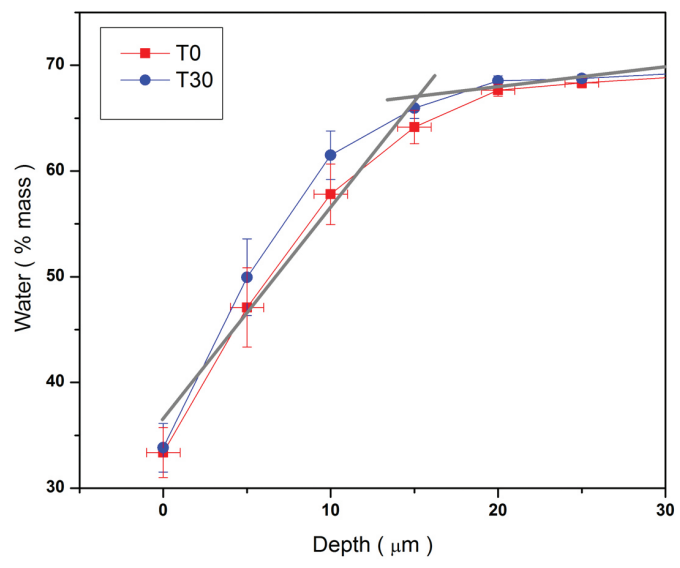

Figure 2. Average water concentration (\% of mass) at different depths for $\mathrm{T} 0$ and $\mathrm{T} 30$. The point of intersection for the gray lines gives an estimate of the stratum corneum thickness.

Figure 3 shows the water content variation between $\mathrm{T} 0$ and T30, which was calculated using Equation (1):

Water $\operatorname{Contet}(\%)=\frac{T 0_{\text {average }}-T 30_{\text {average }}}{T 0_{\text {average }}} \times 100$

For T30, an increase in water content was found with a maximum equal to approximately $6.4 \%$ at a depth of $10 \mu \mathrm{m}$. Between 0 and $15 \mu \mathrm{m}$ in depth is the stratum corneum, which acts as a protective barrier against water loss. 


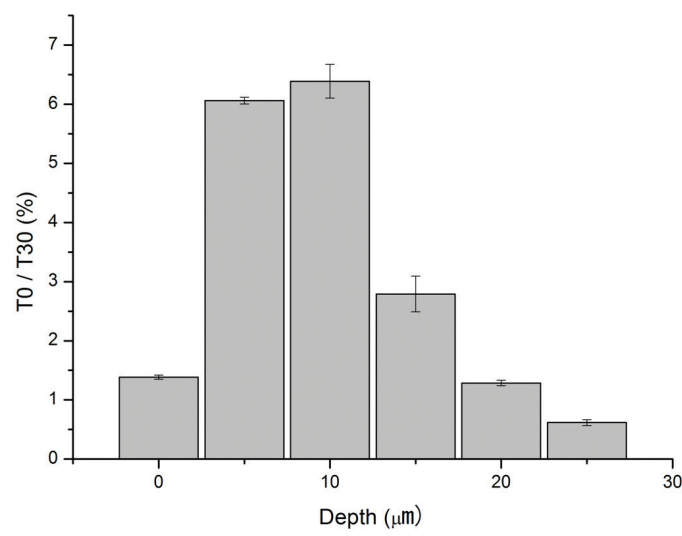

Figure 3. Difference in the average water concentration between T0 and T30.

\section{NMF (Natural Moisture Factor)}

Figure 4 shows the average Raman spectra obtained for the fingerprint region at depths of $0-25 \mu \mathrm{m}$ for $\mathrm{T} 0$ and T30. Using these data, we performed the calculation of NMF concentrations, as described in the methodology.

Figure 5 shows the changes in the NMF content at different depths for $\mathrm{T} 0$ and $\mathrm{T} 30$. As expected for both time points, the amount of NMF decreases at greater depths. However, for T0, the NMF shows practically the same values at 10 and $15 \mu \mathrm{m}$. These results can be correlated with the water concentration in the skin. The semi-quantitative concentration profile of NMF shows an increasing gradient up to $5 \mu \mathrm{m}$ from the skin surface after use of the product as well as an increase in the NMF content from 0.9 to 1.2 (a.u.) at the surface. The calculated NMF average content for $\mathrm{T} 0$ and $\mathrm{T} 30$ was $30.93 \pm 1.09$ (a.u.) at the skin surface and $12.76 \pm 0.48$ (a.u.) at $5 \mu \mathrm{m}$ in depth.

To understand the behavior of the natural moisturizing factor of skin, we compared the levels of two key components of NMF, PCA (pyrrolidone carboxylic acid) and UCA (urocanic acid). Analysis of the in vivo Raman spectra using the spectral model described in the methodology revealed an increase in the amounts of PCA and UCA at the skin surface. Figures $6 \mathrm{a}$ and $\mathrm{b}$ show the average contents of PCA and UCA for volunteers at T0 and T30. For UCA, the increase between $\mathrm{T} 0$ and $\mathrm{T} 30$ was approximately $38.5 \%$ at the skin surface, approximately $1.5 \%$ at $5 \mu \mathrm{m}$ in depth, constant up to $10 \mu \mathrm{m}$, and decreasing thereafter. The PCA content of the NMF showed a small increase of approximately $2.4 \%$ between $\mathrm{T} 0$ and $\mathrm{T} 30$ at the skin surface $(0 \mu \mathrm{m})$, and decreased for greater depths.

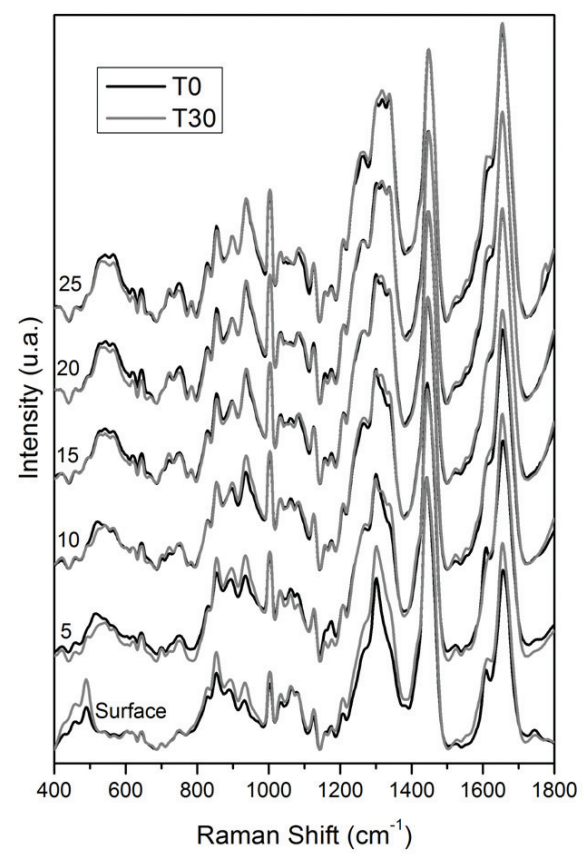

Figure 4. Average Raman spectra T0 (black) and T30 (gray) for different skin depths. For clarification, the spectra have been shifted vertically.

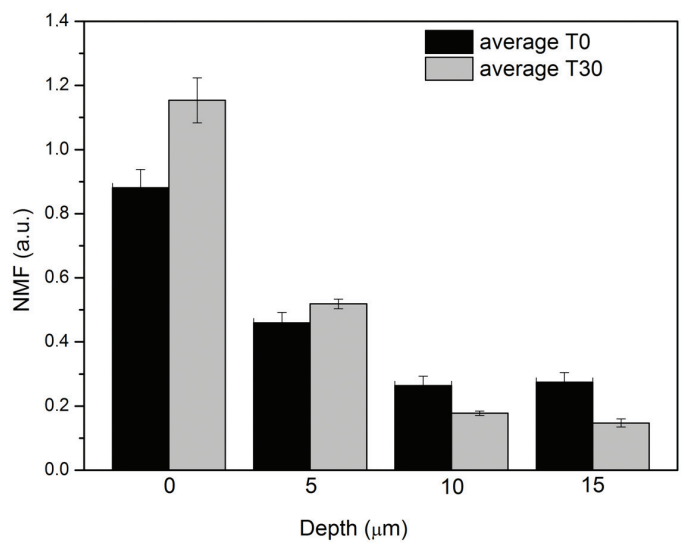

Figure 5. Average NMF concentration for T0 (black) and T30 (gray). The inset shows the semi-quantitative concentration of NMF.

\section{Discussion}

The amount of water content in the skin obtained by confocal Raman spectroscopy for different depths is in agreement with the results in the literature obtained using other techniques, which reports the water content in the stratum corneum to be approximately $70 \%$ (by weight) for healthy skin. The outer skin layer is more susceptible to suffer the aggressions of the environment, such as relative humidity variations. Therefore, these outer layers are largely dependent on ambient humidity and are hydrated to a much lesser degree than deeper 

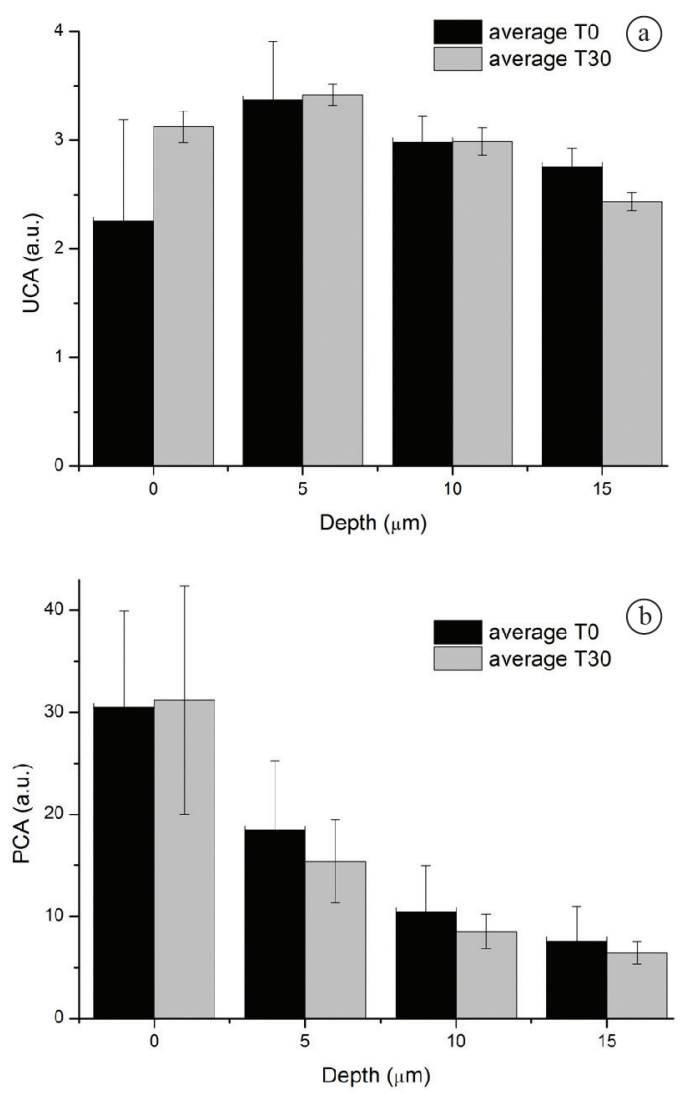

Figure 6. In vivo semi-quantitative concentration of UCA (a) and PCA (b) for the particular region.

layers. In previous work, Raman spectroscopic results showed a water content of approximately $30 \pm 5 \%$ at the skin surface and a continuous increase to $65 \pm 8 \%$ at a depth of approximately $15 \mu \mathrm{m}$ (Caspers et al., 2003). The water content in the deeper layers of the stratum corneum is not easily influenced by changes in external conditions, such as those caused by seasonal variations (Egawa et al., 2007). Normal skin, which has no aspect of dryness, must contain a water content value above $10 \%$, stored both in the intercellular medium and in corneocytes (surface skin cells). Dehydrated skin can present different disorders, including the malfunction of enzymes involved in the desquamation process. These hydrophilic enzymes require water to promote the reactions that will break the cohesive force existing between the corneocytes that are connected by corneodesmosomes bound to protein S. When protease enzymes degrade the protein, the desquamation process occurs. Thus, water acts directly in enzymatic reactions that occur in the stratum corneum, ensuring the functioning and integrity of the tissue (Rawlings and Harding, 2004). Therefore, the significant increase in hydration from 5 to $35 \mu \mathrm{m}$ showed that use of the cosmetic promoted water retention, which is associated with the barrier effect in controlling transepidermal water loss. In the tested product formulation, we found a humidifying active function, which contributes to the retention of water in these layers.

The NMF was increased at the surface and $5 \mu \mathrm{m}$ in depth (Figure 5), which could be related to the hydration at these depths. The NMF is responsible for maintaining the level of hydration of the stratum corneum because it is composed of different hygroscopic molecules. Increases in natural moisturizing factor in the stratum corneum occur when the skin needs water (Wu and Kilpatrick, 2011). The increase in the production of NMF starts with the synthesis of filaggrin from the dephosphorylation of the profilaggrin keratinocytes protein, which is located in the upper layers of viable epidermis. When filaggrin moves to the interface between the stratum granulosum and the stratum corneum, the filaggrin is hydrolyzed (Kezic et al., 2008). This process is confirmed by the content variation in NMF obtained by Raman spectroscopy because it showed a greater increase at the skin surface with a slight decrease to $5 \mu \mathrm{m}$. During the aging process of skin, cell renewal is slower, facilitating the action of hydrolytic enzymes that are responsible for breaking the chains of the filaggrin protein. The disruption of these protein chains results in the release of amino acids and their derivatives, such as pyrrolidone carboxylic acid (PCA) and urocanic acid (UCA), which are responsible for composing the NMF (Bouwstra et al., 2008). Urocanic acid is the result of the metabolic degradation of histidine, an amino acid found in human skin that has a high absorption capacity for UVB rays. However, this component of NMF is water soluble and can be easily removed from the skin surface in different ways, such as perspiration. After 30 days of constant use of the product, an increase in the concentration of UCA in the skin surface was observed.

The methodology of confocal Raman spectroscopy was used to determine the water content and NMF of facial skin in different layers in real time. Using this technique, we found that the cosmetic modified the biochemical composition of the skin by increasing the water concentration and NMF. These changes were positive and suggest mitigation of the effects of skin aging.

\section{References}

Alberti I, Kalia YN, Naik A, Bonny JD, Guy RH. In vivo assessment of enhanced topical delivery of terbinafine to human stratum corneum. Journal of Controlled Release: Official journal of the Controlled Release 
Society. 2001; 71(3):319-27. http://dx.doi.org/10.1016/ S0168-3659(01)00244-9

Bagatin E. Envelhecimento cutâneo e o papel dos cosmecêuticos. Boletim Dermatológico UNIFESP. 2008; 5(17):1-4.

Bergholt MS, Zheng W, Huang Z. Development of a multiplexing fingerprint and high wave number Raman spectroscopy technique for real-time in vivo tissue Raman measurements at endoscopy. Journal of Biomedical Optics. 2013; 18(3):030502. http://dx.doi.org/10.1117/1. JBO.18.3.030502

Bouwstra J, Groenink H, Wouter W, Kempenaar JR, Stefan G. Water distribution and natural moisturizer factor content in human skin equivalents are regulated by environmental relative humidity. Journal of Investigative Dermatology. 2008; 128(2):378-88.

Caspers PJ, Lucassen GW, Bruining H, Puppels GJ. Automated depth-scanning confocal Raman microspectrometer for rapid in vivo determination of water concentration profiles in human skin. Journal of Raman Spectroscopy. 2000; 31:813-8. http:// dx.doi.org/10.1002/1097-4555(200008/09)31:8/9<813::AIDJRS573>3.0.CO;2-7

Caspers PJ, Lucassen GW, Carter E, Bruining H, Puppels GJ. In vivo confocal Raman microspectroscopy of the skin: noninvasive determination of molecular concentration profiles. Journal of Investigative Dermatology. 2001; 116(3):434-42. http://dx.doi.org/10.1046/j.1523-1747.2001.01258.x

Caspers PJ, Lucassen GW, Puppels GJ. Combined in vivo confocal Raman spectroscopy and confocal microscopy of human skin. Biophysical Journal. 2003; 85(1):572-80. http:// dx.doi.org/10.1016/S0006-3495(03)74501-9

Chung JH, Seo JY, Choi HR, Lee MK, Youn CS, Rhie G, Cho KH, Kim KH, Park KC, Eun HC. Modulation of skin collagen metabolism in aged and photoaged human skin in vivo. Journal of Investigative Dermatology. 2001; 117(5):121824. http://dx.doi.org/10.1046/j.0022-202x.2001.01544.x

Costa A. Tratado internacional de cosmecêuticos. Guanabara Koogan; 2012.

De Carvalho LFCS, Sato ET, Almeida JD, Martinho HS. Diagnosis of inflammatory lesions by high-wavenumber FT-Raman spectroscopy. Theoretical Chemistry Accounts. 2011; 130(4-6):1221-9. http://dx.doi.org/10.1007/ s00214-011-0972-2

Djabri A, Guy RH, Delgado-Charro MB. Transdermal iontophoresis of ranitidine: an opportunity in paediatric drug therapy. International Journal of
Pharmaceutics. 2012; 435(1):27-32. http://dx.doi. org/10.1016/j.ijpharm.2012.03.006

Egawa M, Hirao T, Takahashi M. In vivo estimation of stratum corneum thickness from water concentration profiles obtained with Raman spectroscopy. Acta Dermato-Venereologica. 2007; 87(1):4-8. http://dx.doi. org/10.2340/00015555-0183

Fraser SJ, Mulet X, Martin L, Praporski S, Mechler A, Hartley P G, Polyzos A, Separovic F. Surface immobilization of bio-functionalized cubosomes: sensing of proteins by quartz crystal microbalance. Langmuir: ACS Journal of Surfaces and Colloids. 2012; 28(1):620-7. http://dx.doi. org/10.1021/la2032994

Grayson S, Elias PM. Isolation and lipid biochemical characterization of stratum corneum membrane complexes: implications for the cutaneous permeability barrier. Journal of Investigative Dermatology. 1982; 78(2):128-35. http:// dx.doi.org/10.1111/1523-1747.ep12505953

Kezic S, Kemperman PMJH, Koster ES, De Jongh CM, Thio HB, Campbell LE, Irvine AD, Mclean W HI, Mclean IWH, Puppels GJ, Caspers PJ. Loss-of-function mutations in the filaggrin gene lead to reduced level of natural moisturizing factor in the stratum corneum. Journal of Investigative Dermatology. 2008; 128(8):2117-9. http://dx.doi.org/10.1038/ jid.2008.29

Oliveira PK, Tosato MG, Alves RDS, Martin AA. Skin biochemical composition analysis by Raman spectroscopy. Revista Brasileira de Engenharia Biomédica. 2012; 28:27887. http://dx.doi.org/10.4322/rbeb.2012.032

Rawlings AV, Harding CR. Moisturization and skin barrier function. Dermatologic therapy. 2004; 17(1):43-8. http:// dx.doi.org/10.1111/j.1396-0296.2004.04S1005.x

Tosato MG, Alves RS, Dos Santos EAP, Raniero L, Menezes PFC, Belletti KMS, Praes CEO, Martin AA. Raman spectroscopic investigation of the effects of cosmetic formulations on the constituents and properties of human skin. Photomedicine and Laser Surgery. 2012; 30(2):85-91. http://dx.doi.org/10.1089/pho.2011.3059

Wu JQ, Kilpatrick-Liverman L. Characterizing the composition of underarm and forearm skin using confocal Raman spectroscopy. International Journal of Cosmetic Science. 2011; 33(3):257-62. http://dx.doi.org/10.1111/j.14682494.2010.00627.x

Votano JR, Parham M, Hall LH, Kier LB, Hall LM. Prediction of aqueous solubility based on large datasets using several QSPR models utilizing topological structure representation. Chemistry \& Biodiversity. 2004; 1:1829-41. http://dx.doi. org/10.1002/cbdv.200490137

\section{Authors}

Ana Paula Teixeira, João Lucas Rangel, Leandro José Raniero, Priscila Pereira Fávero, Airton Abrahão Martin*

Laboratório de Espectroscopia Vibracional Biomédica, Instituto de Pesquisa e Desenvolvimento - IP\&D,

Universidade do Vale do Paraíba - UNIVAP, Av. Shishima Hifumi, 2911, CEP 12244-000, São José dos Campos, SP, Brasil.

\section{Maira Gaspar Tosato}

Instituto de Química Física de los Materiales, Medio Ambiente y Energía - INQUIMAE,

Universidad de Buenos Aires - UBA, Ciudad Universitaria, Pabellón II, C1428EHA, Buenos Aires, Argentina. 